\title{
REFERENCES
}

Armor, D. J. \& Couch, A. S. Data-text primer: An introduction to computerized social data analysis. New York: Frse Press. London: Collier-Macmillan, 1972.

Cooperband, A. S. Basic TRACE III. CCBS Document TM-6, July 31,1970 .

Cooperband, A. S., Moore, W. H., Jr., Meeker, R. J., \& Shure, G. H. TRACE-III: An implicit programming system for inductive data analysis. Proceedings of the 1971 Annual Conference of the Association for Computing Machinery, 1971, 133-138.

Dixon, W. J. (Ed.) BMD biomedical computer programs. Berkeley and Los Angeles: University of California Press, 1967.

Hunt, E., Marin, J., \& Stone, P. Experiments in induction. New York: Academic Press, 1966.

Meeker, R. J., Shure, G. H., \& Cooperband, A. S. An implementation system for designing computer-based experiments. Proceedings of the 1971 Annual Conference of the Association for Computing Machinery, 1971, 169-178.

Nie, N. H., Bent, D. H., \& Hull, C. H. Statistical package for the social sciences. New York: McGraw-Hill, 1970.

Press, L. I, IDEA: A technique for inductive data exploration and analysis. Unpublished doctoral dissertation, University of
California, Los Angeles, 1967.

Press, L. I., Rogers, M. S., \& Shure, G. H. An interactive technique for the analysis of multivariate data. Behavioral Science, 1969, 14, 364-370.

Shure, G. H., \& Meeker, R. J. Bargaining processes in experimental territorial conflict situations. Peace Research Society (International), 1969, 11, 109-122.

Shure, G, H. \& Meeker, R. J. A computer-based experimental laboratory. American Psychologist, 1970, 25, 962-969.

Shure, G. H., Meeker, R. J., \& Hansford, E. A. The effectiveness of pacifist strategies in bargaining games. Journal of Conflict Resolution, 1965, 9, 106-117.

Shure, G. H., Meeker, R. J., Moore, W. H., Jr., \& Kelley, H. H. Computer studies in bargaining behavior: The role of threat in bargaining. SDC Document SP-2196, 1966, System Development Corporation, Santa Monica, Calif.

Shure, G. H., Press, L. I., \& Rogers, M. S. Man-computer derivations of tree structures from multivariate data. Proceedings of the $76 \mathrm{th}$ Annual Convention of the American Psychological Association, 1968, 3, 215-216.

Sonquist, J. A., Baker, L. B., \& Morgan, J. N. Searching for structure. Ann Arbor, Mich: Braun-Brumfield, 1971.

\section{Project IMPRESS, several perspectives: Interactive computing and data analysis*}

\author{
PAUL VELLEMAN \\ Department of Statistics, Princeton University, Princeton, New Jersey 08540
}

\begin{abstract}
Project IMPRESS is an interactive social science data analysis system used extensively at Dartmouth College and throughout the DTSS network. The programming techniques used to make it an unobtrusive time-sharing job and the user interface design considerations used to make it a system easy for both students and experienced researchers to run are described and their pedagogical and research values discussed.
\end{abstract}

Time-sharing can provide a computing environment ideal for analyzing data and for teaching data analysis. Project IMPRESS is a large interactive data analysis system designed to facilitate both of these activities. In the 5 years since development began, it has become an important part of social science teaching and research at Dartmouth College and has been used widely by the schools and research organizations connected to the Dartmouth Time-Sharing System. ${ }^{1}$

IMPRESS is an acronym for Interdisciplinary Machine Processing for Research and Education in the Social Sciences. It consists primarily of a collection of interrelated programs for data analysis, an on-line archive of over 60 cleaned and processed data sets, and programs for retrieving and reformatting variables selected from these data sets. Provisions are also made for users to clean and process their own data and for communication with user-written data analysis programs.

Perhaps the greatest achievement of Project IMPRESS is the demonstration that such a system can exist: (a) Computer-naive undergraduates use it to learn both

* This work is partially supported by an Atomic Energy Commission contract, No. At-11-1-2310, awarded to the Department of Statistics, Princeton University. social science and data analysis. (Student use accounted for $69 \%$ of the more than 20,000 recorded runs in the past year.) (b) Useful data analysis can be done in little time and at reasonable cost. (The average run in this period took $11 \mathrm{~min}$ and cost $\$ 1.81$ at educational rates.) (c) IMPRESS coexists with other time-sharing users without overtaxing the system. (The Dartmouth Time-Sharing System, DTSS, regularly handles over 140 users simultaneously. ${ }^{2}$ )

These achievements required a careful balancing of conflicting pressures in the design of the user interface and the development of sophisticated data manipulation techniques.

\section{COMPUTING ENVIRONMENT}

The early computers liberated social scientists from the drudgery of mechanical calculators and counting sorters and made possible many techniques previously unthinkable. The interactive computer has the ability to free us from the black box, computer code syntax, one-shot-at-a-time style of working, and to recover one of the advantages of the hand calculator: hands-on data manipulation. It has also permitted the development of 
IMPdess $\quad 15$ OC: 73 09:16

\section{PRIVACY WARWING}

DO YOU WWNY USER'S INSTRUCTIONS? NO

BNTER NAME OF STUDY? GENSOC 73

$$
\begin{aligned}
& \text { General Social survey -- N.O.R.C. } 1973 \\
& N=1504 \text { on } 220 \text { VARIABLES }
\end{aligned}
$$

ENTER NAMES OF 2 TO \& VARLABLES TO BE RUN

$?$ GUNLAW, OWNGUN, GUN, FEAR, SEX, WXYZ, RACE. EDUC, PSUREG

THE FOLLOWING VARIABLE IS INVALID:

WXY 2
RE-ENTER THIS VARIABLE, OR 'DELETE'

? $A G E$

\section{ANALYSIS MODE}

7 EXPLAIN

ENTER 'DISC', 'CONT', OR 'STOP'

ANALYSIS MODE

? DETAIL

\section{ANALYSIS MODES}

DISC -- GENERATES TABLES AND STATISTICS PROM DISCRETE DATA
CONT -- GENERATES VARIANCE-COVARIANCE MATRIX PROM CONTINCONT -- GENERATES VARIANCE-COVARIANCE MATRIX PRON COWTIN

\section{OPT IOAIS}

SAMPLE - QUICKLY OBTAINS A RANDOM SUBSET (SIZE N) OF THE AVATLABLE OBSERVAT IONS IN THE STUDY. LIMIT: 3,000 CASES OR THE SAMPLE SI $2 E$. WHICHEVER IS SMALLER

PORMAT: SAMPLE (N)

FILES - - USER SPECIFIES TWO SCRATCH FILES TO BE USED AS COEMUAICATION PILES

FORMAT: FILES (SCRATCI1, SCRATCH2)

DEFALT: IF NO YTLES ARE SPECIPIED, SCRATCHI AND SCRATCH2 ARC ASSUAED

SIRS-N -- SIMPLE INDEPENDENT RANDOM SAMPLING WITHOUT REPLACEMENT

FORMT: SIRS-N (.5)

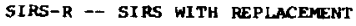

\section{NALYSIB MODX \\ 7. DI8C}

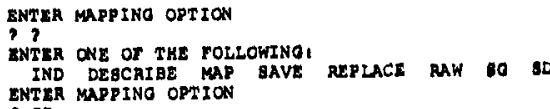

ENTEK COMNAND

7 LIST

$$
\text { AVAILABLE VARIABLES: }
$$

CDUC - R'S EDUCATSOH 2EVEL I 79

SEX FEX OY RESPONDENT! is

MACS - RACS OF REBPONDENT I 73

GUNLAH DOEB R FAVOR OUN iNHE! ?

- HAB R BEEN THREATEHED WITH A OUN:

TEAR I I R ATRID TO WALK IN NEIGIBORHOOD AT NIGHW I

OHNOUN - DOES R HAVE A GUN 13

PBUREO - PAU REOTON I 73

AOE = RESPONDENT'S EXACT AGE I 73
ENTEH COMMAND

7 MAKO I GUNLAW, EXCL

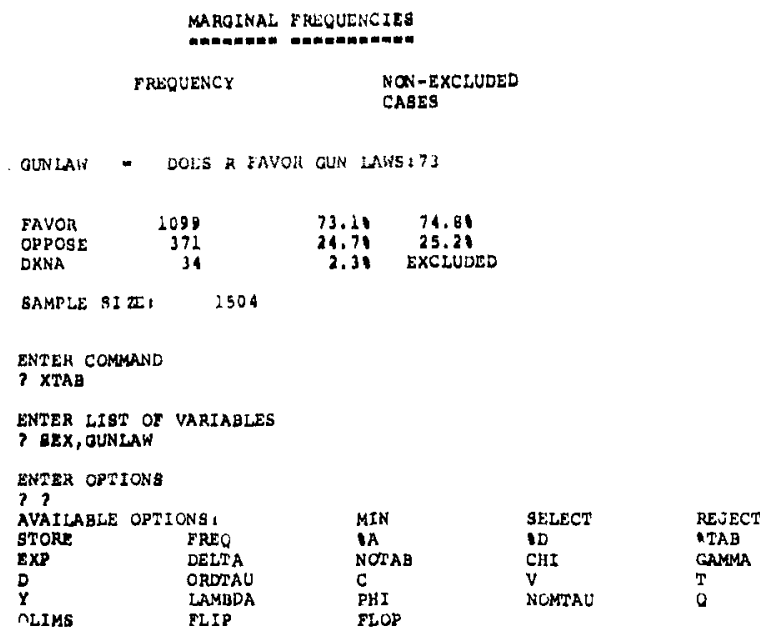

ENTER OPTIONS

? IA

GEX BX GUNLAW

DOWN I, SEX OF REBPONDEN' 173
ACROGS, DOES R SAVOR GUN ENHS:

\begin{tabular}{lrrrr} 
& \multicolumn{2}{c}{ PERCENTAGES ACROBS } \\
& FAVOR & OPPOBE & TOTAL \\
FEHALE & 80.71 & 19.31 & 786 \\
MALE & 68.01 & 32.01 & 684 \\
TOTAL & 74.81 & 25.21 & 1470
\end{tabular}

EXCLUSTON ANALYSIS

TABLE TOTAL! $\quad 1470$

$\begin{array}{lr}\text { EXCLUDEDI } & 34 \\ \text { SAKPLE SI ZE } & 1504\end{array}$

ENTEA COMMAND

XTADISEX,FEAR, IA, CHI, O

BEX BY FEAR

DOW: SEX OF RESPONIJENT 173

ACKOBS, IS R AFRAID TO WALK IN NEIGHBORHOOD AT NIGIT: 73

\begin{tabular}{lrrr} 
& \multicolumn{2}{c}{ PERCENYAGES } & ACROSS \\
& VESIJK & NO & \multicolumn{1}{c}{ TOTAL } \\
TEMALE & 59.69 & 40.21 & 796 \\
MALE & 20.41 & 79.61 & 700 \\
TOTAL & 41.49 & 58.61 & 1496
\end{tabular}

CMI SQUARE - 239.009 WITH $\perp$ D.F

THE PROBAUIIITY OF CHI SOUARE IS $<.00$

YULE'S
0.706

EXCLUSION ANALYSIS

TABLE TOTAL: 1496

EXCLUDEDI
SAMPLE SIZEI $\quad 1504$

I XTAB! 8EX, TEAR, OUNLAN,"

98X by rEAR aY GUNLAW

CONTROLI UEX OF REGPONDENT 13

DOWA, IS A AFRAID TO WALK IN NEIGHMORHOOD AT NIOHTI73 ACROBS: DOS 8 RAVOR OUN LANB 173

esX - reMaLE

Fig. 1. Sample run. 
Figure 1. (continued)
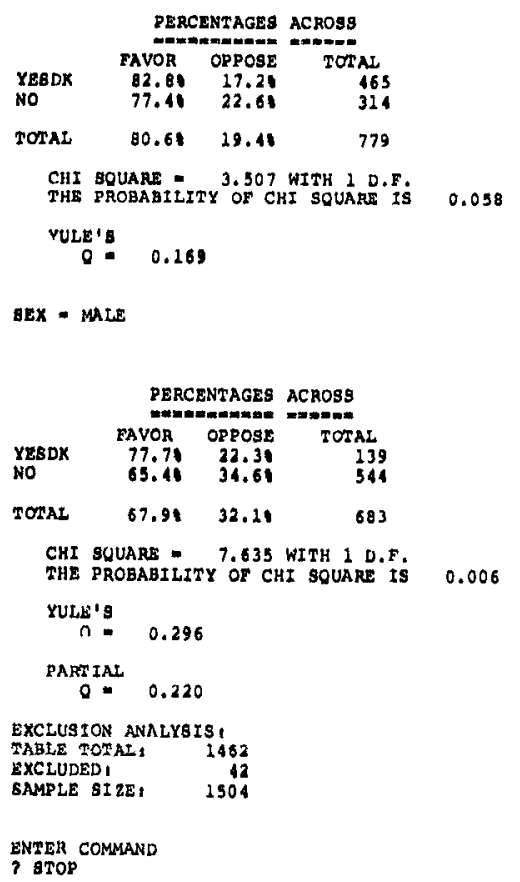

new techniques combining computing power and human guidance.

The way in which a system interacts with users defines the environment in which the data manipulation and analysis are specified. The IMPRESS system creates a computing environment designed to encourage the user to think about his data rather than the program analyzing them. The important aspects of this environment can be summarized as follows: Interactive-Most commands receive a response in less than $4 \mathrm{sec}$, many instantly, even with a full user load on the system. Data analysis algorithms requiring frequent user decisions fit naturally into the command structure. Conversational-All commands and options have mnemonic names, all variables have names and descriptions, and all responses are easy to read and interpret. The output from a run can be read without reference to a manual or code book. Protected-An illegal command receives an informative error message and does no harm. Explanations of correct responses are available from the system at any time. Structured-While there is complete freedom to move among the statistical routines, there is a structure where needed to guide users. The data retrieval and remapping routines form a separate sequence which must be followed in order, and several of the statistical routines have their own structure dictated by the logic of the data analysis method. Succinct-An experienced user can specify commands and options in a single line. A less experienced user can specify one at a time, awaiting a prompt for each. A beginning user has explanations available at each step. Where it is meaningful, the option DITTO, or ", calls for the use of the option previously specified. This design avoids unnecessary printing and reduces the frequency of turn-around-important considerations when many users are limited to 10-character/sec terminals.

The easiest way to describe the IMPRESS computing environment is to demonstrate its important aspects. A sample run is shown in Fig. 1 using data collected recently by NORC as part of a long-term study of social attitudes in the United States.

This run was generated to drive the major features of the IMPRESS user interface. It demonstrates recovery from user errors, the different levels of explanation available from the system, and both prompted and concise command specifications. It should be noted that all of these features are available in the appropriate forms throughout a run.

The versatility of interactive computing offers a range of environment designs. At one end is the "black box" program which receives data and returns a completed analysis. Its internal workings are hidden from the user, and with them its assumptions, default decisions, and often its treatment of extreme or missing data points. Output from the black box is often useful and sometimes meaningless-but it won't tell us which. At the other end of the scale is the totally versatile "transparent box" environment, such as that provided by APL. To control its myriad features, one must learn a new language and invest programming time. With this freedom comes responsibility; there is little hope of protection from command errors, and data manipulations can become awkward.

Between these extremes is the "translucent box" system. It protects the user from the dangers of command errors and painlessly provides clear data and properly labeled output. It speaks and reads an English-like language which it explains on request. While performing major calculations out of view, it reports regularly, giving users the impression of watching the analysis and allowing or requiring their intervention at key decision points.

Translucent box systems vary widely in the restrictiveness of their command sequences and the visibility of their computations. IMPRESS has been forced by its pedagogical obligations to be perhaps less transparent than one might wish. It does, however, give one freedom to choose analysis techniques and the feeling of working with hands on the data.

\section{INTERNAL DESIGN}

The internal structure of IMPRESS has been carefully developed to minimize strain to the Dartmouth Time-Sharing System. Impress is written entirely in Dartmouth BASIC. ${ }^{3}$ This choice was made to take advantage of the file manipulation, string handling, and program segmentation features of BASIC not available in other languages at that time. It has, unfortunately, limited the exportability of the complete package. 
Table 1

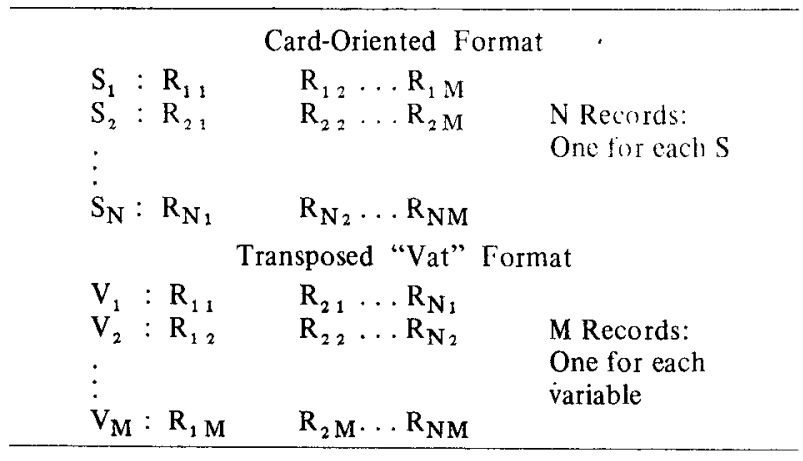

However, many of the techniques developed for IMPRESS have general usefulness.

Data management is a major part of the work IMPRESS performs. Large data transfers strain the capabilities of a time-sharing system. If IMPRESS dealt with standard format data matrices, the data transfers alone would make it impractical. DTSS constrains programs in general use to occupy no more than $16 \mathrm{~K}$ words (equivalent to $72 \mathrm{~K}$ bytes) of core. This would barely be room for a large data matrix, yet the IMPRESS archive includes the 1970 Census Neighborhood Characteristics Public Use 1 in 10,000 sample $(20,361$ observations on 80 variables) and economic time series data from the Brookings Institute (96 observations on 2,477 variables).

Several techniques have been used to reduce data transfers. The most important of these is the use of transposed data files. Standard data matrices have one record for each $S$, with codes for each variable appearing in order. This is natural for data stored on cards, but it forces a data analysis program to read the entire data matrix for a variable to be selected. IMPRESS data files are arranged so that each record is a "vat" of observations on a single variable (Table 1). Each vat must have an entry for each $\mathrm{S}$ in a consistent order. This simplifies the selection of a variable from a stored data set since IMPRESS need only locate the appropriate vat--a trivial task when a directory exists and addressable random access files are used.

To further minimize data transfers, IMPRESS files are packed more densely than normal. An extension of the CHANGE statement in BASIC provides programmer control over each bit in a string. Since most data sets are still coded with cards in mind, the 4-bit half-byte, or nibble, was chosen as the unit of storage; its 16 possible states will hold all the information in a singly punched column.

Once the data have been retrieved by IMPRESS and restructured according to user instructions, they are again reduced. Several techniques are used, chosen according to the type of data used. The most interesting and original of these is the use of mixed-radix positional numbers. In any positional number system, each position has a certain number of states. We are used to number systems where each position has the same number of states-10 in decimal, 2 in binary, 16 in hexadecimal--but the logic of positional numbers doesn't require a single radix. In any positional system, each position counts at a fixed rate-for example, 1 , $10 \mathrm{~s}, 100 \mathrm{~s}$, etc., in decimal. This rate is the product of the number of states for all the positions to the right which in a single radix system yield successive powers of the radix. The reduction of codes on several variables is accomplished by converting the appropriate mixed radix number into its decimal equivalent. Since this scheme counts codes from 0 , variables are recoded in IMPRESS to start at 0 so that no categories are wasted.

To obtain the original codes from a mixed radix number, we must know the order in which the variables were coded and the number of categories in each. To recover the value of any particular variable, one shifts the mixed radix positional number to the right by dividing by the counting rate for the position desired, discarding the remainder, and taking the quotient modulo the number of categories in the variable.

\section{SPECIAL OPTIONS}

There are two parts of IMPRESS that increase its versatility greatly. The variable construction system provides a complete logical compiler for discrete variables and all of the algebraic expressions and special functions of BASIC for continuous variables to make the definition of new variables as natural as possible. New variables may be constructed from the existing variables in a data set to create scales or define new categories. An assortment of simulation routines allows the generation of dummy variables with predetermined attributes. This is especially useful for giving beginning statistics students samples guaranteed to fit the assumptions of classical methods with known population parameters. One short exercise can, for example, make the effect of increasing the sample size on a confidence interval more

Table 2

Coding Data Into Mixed Radix Positional Numbers for a Subject Given His Sex, Race, Age, Income, and Religion Where These Variables Have Been Coded Into Discrete Categories

Mixed Radix Positional Numbers

\section{Constant Radix}

\#Counts

Rates

Mixed Radix

Variables

\#Categories

Rates (Product Vector)

Values to Code

Mixed Radix Number

Decimal Equivalent

$\begin{array}{rcccc}10 & 10 & 10 & 10 & \\ 1000 & 100 & 10 & 1 & \\ \text { Relig- } & \text { In- } & & & \\ \text { ion } & \text { come } & \text { Age } & \text { Race } & \text { Sex } \\ 3 & 4 & 5 & 4 & 2 \\ 160 & 40 & 8 & 2 & 1 \\ 1 & 0 & 3 & 2 & 1 \\ 10321 & & & & \\ 1 * 160+0 * 40+3 * 8+2 * 2+1 * 1=205\end{array}$

Translation

To obtain the code for age from this number, the element of the product vector for age is 8

$\operatorname{INT}(205 / 8)=28$

28 modulo $5=3$ which was the age code 


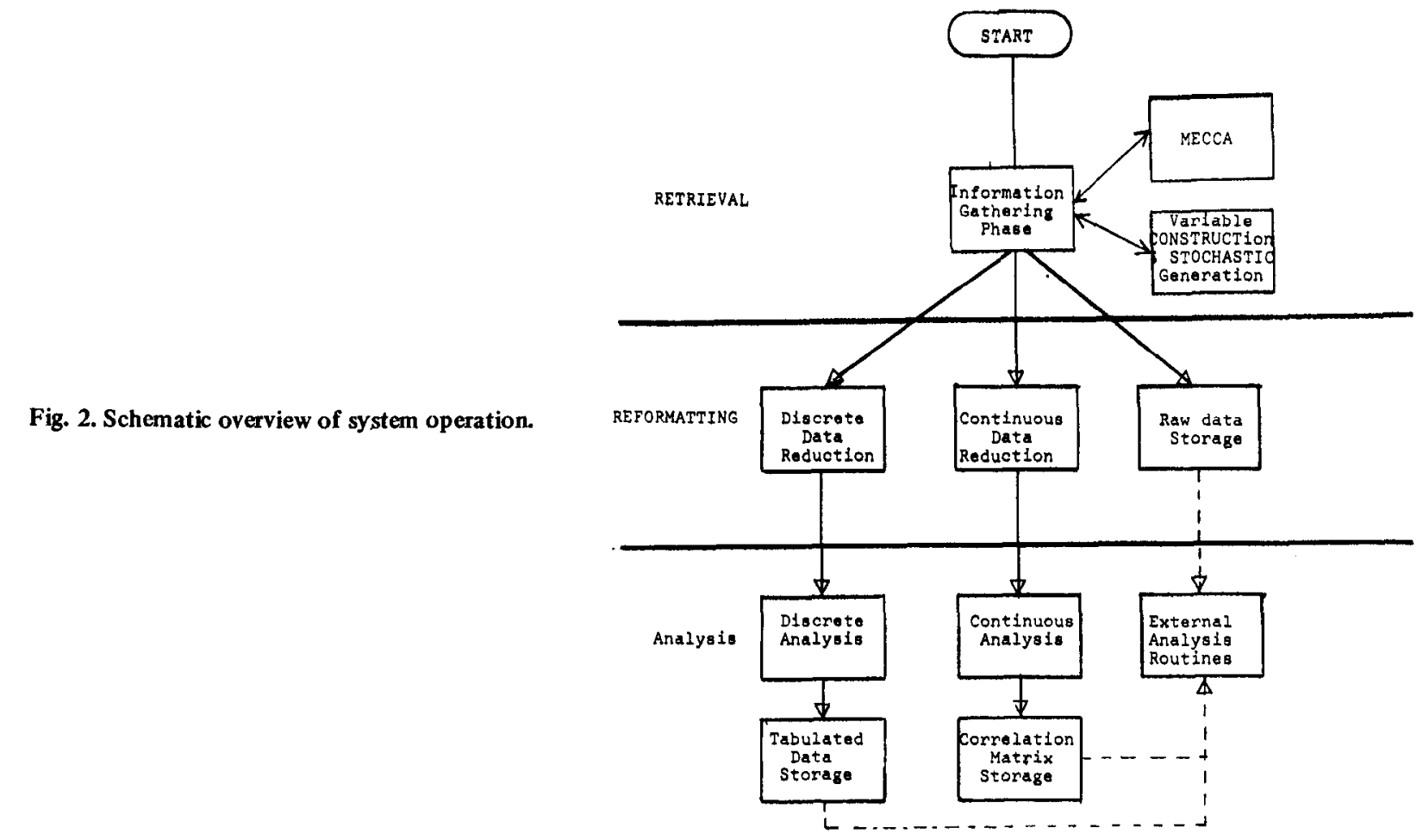

understandable than additional time spent on the relevant algebra.

The MECCA system (Maximum Extensibility for Cross Cultural Analysis) was originally designed for anthropologists, but it provides a powerful tool for the analysis of any matched data sets. MECCA uses a variable in each cross-cultural study coded to uniquely identify any of 1,700 cultures to match data on the same culture from different studies. This permits the construction of data sets previously out of reach of most researchers. For example, frequently anthropologists in the field concentrate on a narrow range of variables. With MECCA, the large collection of variables in Murdock's Ethnographic Atlas are available to be added to a sparser data set. It is possible, of course, to apply MECCA to any collection of data sets concerning the same population, provided each $\mathrm{S}$ has been assigned a unique identification code.

The preparation of unprocessed data for IMPRESS is aided by a separate set of programs in which the data are cleaned and checked for inconsistencies. The user must supply mnemonic names and descriptions for variables and categories. It is possible to code a few variables at a time since newly processed variables can be easily appended to the transposed data file. If the structure of the data set-the number of categories in discrete variables or the range of continuous variables-is known, a file containing the necessary information can be built even before the data are available to speed processing. Surveys of Dartmouth students have been on-line within a week of the return of the questionnaires. Experimental research data can be available even more quickly if data are collected on-line.

No data analysis system can ever be complete. New methods will be developed, and data sets requiring unusual methods will need analyzing. IMPRESS provides the opportunity to store either a transposed data file or a standard data matrix in a user's private file. This file is readily available to independently written data analysis programs. Thus, the data archive, variable construction, and data reformatting functions of IMPRESS can be harnessed by users who wish to use their own analysis programs.

One collection of programs intended for such use was developed by the author to implement several of John Tukey's data analysis proposals. At present, a versatile six-line plotter (Andrews \& Tukey, 1973), a collection of nonlinear data smoothers, and a "median polish" two-way table decomposition (Tukey, in press) are available. A more extensive package of exploratory techniques using IMPRESS as a data management system will be developed if adequate financial support can be found.

Project IMPRESS has had a profound effect on the teaching and, more importantly, on the learning of data analysis at Dartmouth. Many social science students first see real data in graduate school after digesting theories and struggling through a statistics course. At Dartmouth, freshmen and sophomores learn how data behave through experience by doing their own analyses. When they are then taught theories, they are prepared to support or challenge them with facts. When they learn statistics, it is with an idea of its uses and usefulness. In addition, they are introduced to the computer in a protected environment. Frequently, experience with IMPRESS provides the motivation to learn sophisticated programming techniques.

John Kemeny has proposed that the computer should 
be thought of as an education utility in much the same way that we regard the university library. This attitude has developed at Dartmouth. It is as common for some professors to lell a class to learn about, for example, socio-economic status using IMPRESS as to assign reserved collection readings. As the data archive rrows and as more peripheral special-purpose programs are developed, IMPRESS promises to play a growing role in social science research and education.

\section{REFERENCES}

Andrews, D. \& Tukey, J. W. Teletypewriter plots for data analysis can be fast: 6 -line plots, including probability plots. Journal of the Royal Statistical Society Series C, 1973, 22 . 192-202.

Cline, H. F., \& Meyers, E. D., Jr. Problem-solving computer systems for instruction in sociology. American Sociologist. $1970,5,365-370$.

Davis, J. A. Using the IMPRESS system to teach sociology. In Proceedings of the Conference on Computers in the Undergraduate Curricula. Hanover, N.H: University Press of New England, 1971. Pp. 382-387.

Meyers, E. D., Jr. Interactive systems and social science research and instruction. Social Sciences Information, 1970, 9, 157-171.

Meyers, E D Jr. Time-sharing computation in the social sciences. Englewood Cliffs, N.J: Prentice-Hall, 1973.

Tukey, J. W. Exploratory data analysis. Reading, Mass: Addison Wesley, in press.

\section{NOTES}

1. The DTSS network extends south to New Jersey, north to Canada, and west to Illinois. It can also be dialed up directly from anywhere in the United States on a pay-as-you-go basis. Computer power is also wholesaled to NERComP (New England Regional Computer Program) and resold to about 40 colleges and universities in northern New England.

2. A check on a typical weekday showed a maximum of 143 simultaneous users and a total, by 17:00, of 1,926 users logged on during the previous $9 \mathrm{~h}$.

3. Dartmouth BASIC Sixth Edition. A precise description of the language can be found in TM028: "BASIC: A specification," S. Garland, February 1973, available from the Kiewit Computing Center, Hanover, N.H. 03755.

4. Chapin, D. A.. \& Meyers, E. D., Jr. Project IMPRESS: An interactive social science software package. In Robert $F$. Hargraves, Jx. (Ed.), Effective applications of time-sharing at Dartmouth College. In preparation. 